\section{The autoregulated instability of Polo-like kinase 4 limits centrosome duplication to once per cell cycle}

\author{
Andrew J. Holland, ${ }^{1,5}$ Daniele Fachinetti, ${ }^{1,4}$ \\ Quan Zhu, ${ }^{2,4}$ Manuel Bauer, ${ }^{3,4}$ Inder $M$. Verma, ${ }^{2}$ \\ Erich A. Nigg, ${ }^{3}$ and Don W. Cleveland ${ }^{1}$ \\ ${ }^{1}$ Ludwig Institute for Cancer Research, Department of Cellular \\ and Molecular Medicine, University of California at San Diego, \\ La Jolla, California 92093, USA; ${ }^{2}$ Laboratory of Genetics, The \\ Salk Institute for Biological Studies, La Jolla, California 92037, \\ USA; ${ }^{3}$ Biozentrum, University of Basel, 4056 Basel, Switzerland
}

Centrioles organize the centrosome, and accurate control of their number is critical for the maintenance of genomic integrity. Centriole duplication occurs once per cell cycle and is controlled by Polo-like kinase 4 (Plk4). We showed previously that Plk4 phosphorylates itself to promote its degradation by the proteasome. Here we demonstrate that this autoregulated instability controls the abundance of endogenous Plk4. Preventing Plk4 autoregulation causes centrosome amplification, stabilization of p53, and loss of cell proliferation; moreover, suppression of p53 allows growth of cells carrying amplified centrosomes. Plk4 autoregulation thus guards against genome instability by limiting centrosome duplication to once per cell cycle.

Supplemental material is available for this article.

Received September 27, 2012; revised version accepted November 14, 2012.

Centrosomes are microtubule-organizing centers (MTOCs) comprised of a pair of microtubule-based structures termed centrioles, surrounded by proteinaceous pericentriolar material (PCM) (Gonczy 2012). Centrioles, the organizer of the centrosome, normally duplicate once per cell cycle before the onset of mitosis to create two copies of the centrosome to instruct the formation of the bipolar mitotic spindle apparatus (Tsou and Stearns 2006). Abnormalities in centriole duplication can result in the production of extra copies of centrosomes (known as centrosome amplification), which can lead to errors in mitotic spindle formation and chromosome missegregation (Ganem et al. 2009; Silkworth et al. 2009). Centrosome amplification is frequently observed in aneuploid human tumors, where it is proposed it promotes genome instability and tumor development (Nigg and Raff 2009; Holland and Cleveland 2012). Therefore, accurate control

[Keywords: mitosis; aneuploidy; SCF; centrosome; centriole; genome instability; p53]

${ }^{4}$ These authors contributed equally to this work.

${ }^{5}$ Corresponding author

E-mail a1holland@ucsd.edu

Article is online at http://www.genesdev.org/cgi/doi/10.1101/gad.207027.112. of centrosome number is critical for the maintenance of genomic integrity.

Centriole duplication is under the control of Polo-like kinase 4 (Plk4; also known as SAK in Drosophila), a conserved upstream regulator of centriole assembly (Bettencourt-Dias et al. 2005; Habedanck et al. 2005). Loss of Plk4 results in a failure to assemble new centrioles, while, conversely, overexpression of the kinase drives the assembly of excessive new centrioles and subsequent centrosome amplification (Bettencourt-Dias et al. 2005; Habedanck et al. 2005; Kleylein-Sohn et al. 2007; Peel et al. 2007). Abnormal expression of Plk4 has been linked with genomic instability and a predisposition to tumorigenesis (Macmillan et al. 2001; Ko et al. 2005; Basto et al. 2008; Liu et al. 2012), and thus Plk4 abundance must be tightly regulated in order to correctly control centrosome number and maintain genome integrity (Holland et al. 2010a). Identifying the pathways responsible for controlling Plk4 abundance is important for our understanding of centriole duplication and the origin of centrosome amplification in cancer.

Plk4 is a low-abundance kinase whose stability is directly linked to the activity of the enzyme, with active Plk4 phosphorylating itself to promote its own destruction through the ubiquitin-proteasome pathway (Holland et al. 2010b; Brownlee et al. 2011). The SCF (Skp/Cullin/ F-box) E3 ligase associates with phosphorylated Plk4 through the F-box protein $\beta$-TrCP (known as Slimb in Drosophila). Phosphorylation of two residues within the $\beta$-TrCP-binding motif of Plk4 promotes the binding of $\beta$-TrCP and subsequent ubiquitylation and destruction of the kinase (Cunha-Ferreira et al. 2009; Rogers et al. 2009; Guderian et al. 2010; Sillibourne et al. 2010). In Drosophila, the phosphatase PP2A in complex with its targeting subunit, Twins (known as B/PR55 in humans), counteracts Plk4-mediated autophosphorylation to stabilize Plk4 during mitosis (Brownlee et al. 2011).

Despite the recent progress in our understanding of centriole duplication, all of the work to date studying Plk4 regulation has relied on overexpressed Plk4 transgenes. Therefore, it is not established to what extent autocatalyzed destruction controls the stability of endogenous Plk4 and whether autoregulation plays a crucial role in controlling normal centriole duplication. In this study, we used gene targeting in human cells to determine the importance of the autoregulated destruction of Plk4 in controlling centrosome copy number.

\section{Results and Discussion}

To establish the importance of Plk4 self-regulation, we used gene targeting in nontransformed, telomerase-immortalized, human RPE-1 cells to create knock-in alleles of Plk4 that are predicted to be deficient in self-regulation. Since loss of Plk4 autoregulation is predicated to have a dominant effect that may be detrimental to the long-term viability of cells, we used homologous recombination to create heterozygous, conditional alleles of Plk4 by inserting a transcriptional/translational stop cassette into intron 5 of the endogenous Plk4 locus (Supplemental Fig. S1A). The transcriptional/translational STOP prevents transcription and truncates any translation downstream from exon 5 of the Plk4 gene, thereby 
creating a null allele (Holland et al. 2012). However, this cassette is flanked by LoxP sites and can be excised by Cre recombinase to restore expression of the targeted allele.

Homologous recombination was used to create cell lines containing conditional Plk $4{ }^{\mathrm{WT}}$ (wild-type), $\mathrm{Plk} 4^{\mathrm{AA}}$, and Plk $4^{\Delta 24}$ alleles integrated at the endogenous genomic locus (Supplemental Fig. S1A): The Plk4 ${ }^{\mathrm{AA}}$ allele contains mutations in both of the phosphorylation sites required for $\beta$-TrCP binding to Plk4, while the Plk $4^{\Delta 24}$ allele contains a deletion of a 24-amino-acid region that is rich in serine/threonine residues and heavily autophosphorylated (Supplemental Fig. S1B; Cunha-Ferreira et al. 2009; Rogers et al. 2009; Guderian et al. 2010; Holland et al. 2010b). This 24-amino-acid domain contains a conserved binding motif for SCF- $\beta-\operatorname{TrCP}$ and is required for self-catalyzed destruction of kinase-active Plk4 (Holland et al. 2010b).

In the basal state, cells heterozygous for the conditional $\mathrm{Plk} 4^{\mathrm{WT}}, \mathrm{Plk} 4^{\mathrm{AA}}$ and $\mathrm{Plk} 4^{\Delta 24}$ alleles (hereafter referred to as $\mathrm{Plk} 4^{+/ \mathrm{WT}}, \mathrm{Plk}^{+/ \mathrm{AA}}$, and $\mathrm{Plk} 4^{+/ \Delta 24}$ cells, respectively) displayed a $50 \%$ reduction in Plk4 mRNA (Holland et al. 2012) but have normal centrosome numbers and growth rates (Fig. 1E-H). Using quantitative immunofluorescence, we determined the level of Plk4 at the centrosome before and after activation of Plk4 conditional alleles with Cre recombinase. The Plk $4^{\mathrm{WT}}$ conditional allele forms an important control for any effects derived from expression of Cre in cells. To allow for continued growth (see below), we analyzed Plk4 levels (Fig. 1A-C) in cells expressing the SV40 large T-antigen. In the basal state, $\mathrm{Plk} 4^{+/ \mathrm{WT}}, \mathrm{Plk}^{+/ \mathrm{AA}}$, and Plk4 $4^{+/ \Delta 24}$ cells accumulated similar levels of Plk4 at the centrosome, and this level was unchanged following activation of the Plk $4{ }^{\mathrm{WT}}$ allele with Cre recombinase (Fig. 1A,B). In contrast, activation of either of the Plk4 alleles that are deficient in autoregulation resulted in a sixfold to eightfold increase in centrosome-associated Plk4 (a 6.3-fold increase for Plk4 ${ }^{+/ A A}$ cells and an 8.4-fold increase for Plk $4^{+/ \Delta 24}$ cells). Since only a portion of the total Plk4 pool is likely to be associated with the centrosome at a given time, we determined the total number of Plk4 molecules per cell using protein absolute quantification (AQUA) mass spectrometry (Gerber et al. 2003). For this, a known amount of a synthetic "heavy" labeled standard Plk4 peptide was introduced into cell lysates. Protease-digested lysates were then analyzed by tandem mass spectrometry, and the internal standard peptide was used to determine the absolute level of the corresponding native Plk4 peptide. RPE1 cells contained, on average, 3730 ( \pm 986$)$ molecules of Plk4 per cell, a value very similar to that observed in cells expressing the Plk $4{ }^{\mathrm{WT}}$ allele $(3670[ \pm 620]$ molecules per cell) (Fig. 1C). On the other hand, loss of Plk4 autoregulation resulted in a fivefold to sixfold increase in the number of Plk4 molecules per cell $(22,400$ [ \pm 3340$]$ molecules for Plk4 $4^{+/ A A}$ cells, and 20,300 [ \pm 4080$]$ molecules for Plk4 $4^{+/ \Delta 24}$ cells). While activation of the Plk $4^{\mathrm{WT}}$ allele had no effect on centrosome number, the increased Plk4 level from loss of Plk4-autoregulated destruction in $\mathrm{Plk} 4^{+/ \mathrm{AA}}$ and Plk $4^{+/ \Delta 24}$ cells resulted in dramatic centrosome amplification, with $>80 \%$ of these cells possessing more than two centrosomes within $2 \mathrm{~d}$ after viral transduction to express Cre (Fig. 1E). Activation of the Plk4 ${ }^{\mathrm{AA}}$ and $\mathrm{Plk} 4^{\Delta 24}$ allele caused very similar effects in cells, demonstrating that, of the 13 potential autophosphorylation sites contained within the 24-amino-acid Plk4 phos-
A
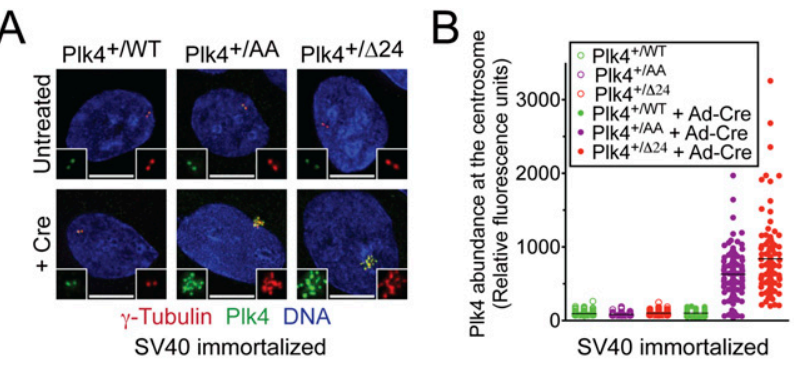

C
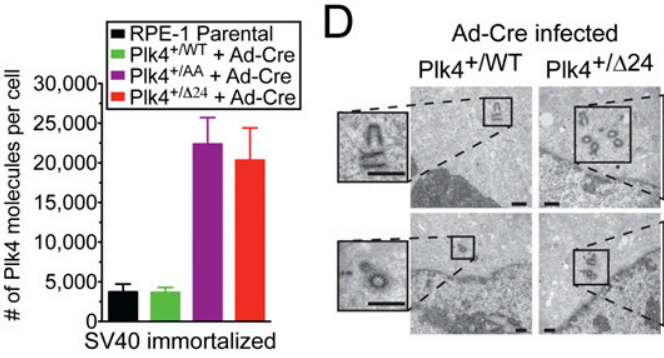

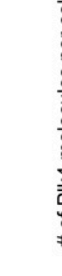

$\mathrm{E}$

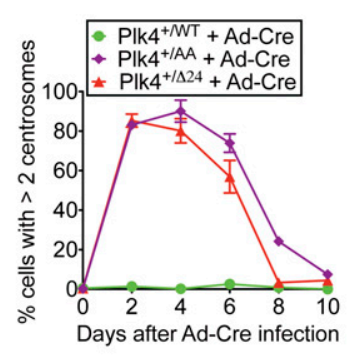

$\mathrm{F}$

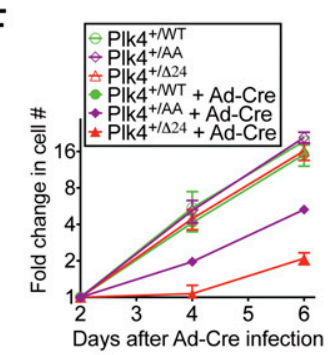

G

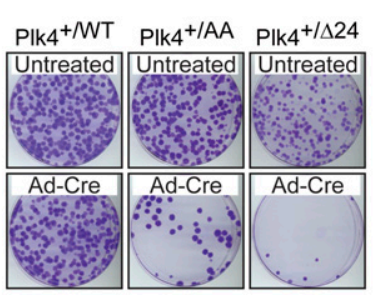

$\mathrm{H}$

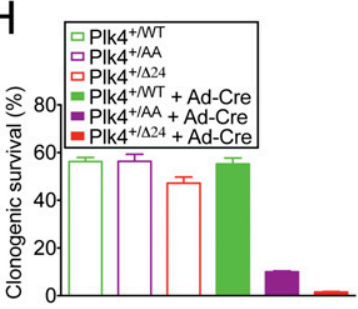

Figure 1. The autoregulated destruction of Plk4 limits centrosome duplication to once per cell cycle. To allow for continued cell growth, cells analyzed in $A-C$ express the SV40 large T-antigen; cells in $D-H$ did not. (A) Images show the level of Plk4 at the centrosome. (Red) $\gamma$-Tubulin; (green) Plk4; (blue) DNA. Bar, $5 \mu \mathrm{m}$. (B) Quantitative immunofluorescence analysis of Plk4 levels at the centrosome. Points show the fluorescence intensity of individual cells from at least two independent experiments. The horizontal line represents the mean. (C) The number of Plk4 molecules per cell was determined using AQUA mass spectrometry. Bars represent the mean of at least two replicas. Error bars represent the standard deviation (SD). (D) Thin-section transmission electron micrographs of cells $2 \mathrm{~d}$ after infection with adenoviral Cre (Ad-Cre). Bar, $0.5 \mu \mathrm{m}$. (E) Fraction of cells with more than two centrosomes at various times after infection with Ad-Cre. Points show the mean of at least three independent experiments. Error bars represent the standard error of the mean (SEM). $(F)$ Fold increase in cell number at various times starting $2 \mathrm{~d}$ after infection with Ad-Cre. Points show the mean of at least three independent experiments. Error bars represent the SEM. (G) Images of crystal violet-stained colonies. $(H)$ Percent congenic survival of the indicated cells. Bars represent the mean of at least three independent experiments carried out in triplicate. Error bars represent the SEM.

phodegron (Holland et al. 2010b; Sillibourne et al. 2010), phosphorylation of the two sites required for $\beta$-TrCP binding (S285 and T289) plays the dominant role in 
controlling Plk4 stability. Rosette-like arrangements of procentrioles were frequently observed $24 \mathrm{~h}$ after expression of Cre in Plk $4^{+/ \Delta 24}$ cells, suggesting that centrosome amplification occurs via the canonical centriole duplication pathway in cells lacking Plk4 autoregulation (Supplemental Fig. S1C). Electron microscopy revealed that the excessive centrioles generated in cells deficient in Plk4 selfregulation had a normal ultrastructure (Fig. 1D). This evidence establishes that self-catalyzed destruction of Plk4 plays not only an important role in controlling endogenous protein levels, but also a crucial role in restricting centriole duplication to allow the production of a single new centrosome per cell cycle.

We used our gene targeted cell lines to examine the primary effect of loss of Plk4 autoregulation in nontransformed human cells. The dramatic increase in centrosome amplification in $\mathrm{Plk} 4^{+/ \mathrm{AA}}$ and $\mathrm{Plk} 4^{+/ \Delta 24}$ cells by $2 \mathrm{~d}$ post-Cre expression was followed by a progressive decline in the proportion of cells with extra centrosomes (Fig. 1E): By $10 \mathrm{~d}$ after Cre expression, $<10 \%$ of Plk4 $4^{+/ A A}$ and Plk4 $4^{+/ \Delta 24}$ cells exhibited centrosome amplification. Since our adenoviral transduction is only $\sim 90 \%$ efficient, we reasoned that the decline in cells with centrosome amplification may be due to proliferation deficits in the cells with extra centrosomes that permit them to be outcompeted by untransduced cells. Consistently, the growth rate of $\mathrm{Plk} 4^{+/ \mathrm{AA}}$ and Plk $4^{+/ \Delta 24}$ cells was dramatically reduced following Cre expression, while the growth properties of Plk4 $4^{+/ W T}$ cells were unchanged (Fig. 1F).

The long-term growth potential of cells that have lost autoregulated control of endogenous Plk4 levels was determined in a clonogenic survival assay. Expression of Cre in Plk4 ${ }^{+/ A A}$ and Plk $4^{+/ \Delta 24}$ cells dramatically reduced colony formation, while Cre expression in Plk4 ${ }^{+/ \mathrm{WT}}$ cells had no effect (Fig. 1G,H). Moreover, the few surviving colonies in Cre-infected $\mathrm{Plk} 4^{+/ \Delta 24}$ cells were analyzed by PCR and found to have escaped Cre-mediated recombination (18 of 18) (data not shown). Therefore, autoregulated control of endogenous Plk4 levels is essential for long-term cell growth.

To verify our observations, we created an additional pair of targeted RPE-1 cells carrying conditional Plk 4 WT and Plk $4^{\Delta 24}$ knock-in alleles in which the transcriptional/ translational stop cassette was inserted into intron 4 (as opposed to intron 5) of the endogenous Plk4 gene (Supplemental Fig. S2A). Again, activation of the Plk $4^{\Delta 24}$ allele resulted in an increase ( $\sim 4.2$-fold) in centrosome-associated Plk4 (Supplemental Fig. S2B,C), centrosome amplification (Supplemental Fig. S3A-C), impaired proliferation (Supplemental Fig. S3D-F), and a dramatically reduced clonogenic survival (Supplemental Fig. S3G,H).

To determine the effect that activation of the $\mathrm{Plk}_{4}{ }^{\Delta 24}$ allele had on cell division, we created $\mathrm{Plk} 4^{+/ \mathrm{WT}}$ and $\mathrm{Plk} 4^{+/ \Delta 24}$ cells coexpressing EYFP- $\alpha$-tubulin and histone $\mathrm{H} 2 \mathrm{~B}-\mathrm{mRFP}$ and monitored cells by time-lapse micros- copy. Filming began $1 \mathrm{~d}$ after transduction with adenoviral Cre and proceeded for $12 \mathrm{~h}$. During this time, all of the Plk $4^{+/ \mathrm{WT}}$ cells divided normally, while $36 \%$ of the $\mathrm{Plk} 4^{\Delta 24}$-expressing cells divided abnormally with an increased mitotic duration (average of $22 \mathrm{~min}$ for normal divisions compared with $35 \mathrm{~min}$ for abnormally dividing cells) (Fig. 2A-C; Supplemental Movie S1). Newly assembled centrioles undergo a modification during mitosis that allows them to recruit PCM and act as an MTOC in the next cell cycle (Wang et al. 2011). The low frequency of abnormally dividing Plk $4^{+/ \Delta 24}$ cells at $1 \mathrm{~d}$ after Cre expression is likely to reflect the additional time required for the newly assembled centrioles to mature into MTOCs.

All abnormally dividing Plk4 $4^{+/ \Delta 24}$ cells entered mitosis with more than two MTOCs, and in most instances $173 \%$ of the time), cells failed to cluster these into two groups prior to division, dividing in a multipolar fashion (Fig. 2A,B; Supplemental Movie S2). The remaining abnormally dividing Plk $4^{+/ \Delta 24}$ cells coalesced multiple MTOCs into two groups prior to anaphase and divided in a bipolar fashion (Fig. 2A,B; Supplemental Movie S3). Remarkably, $2 \mathrm{~d}$ after transduction with adenoviral Cre, mitotic divisions in $\mathrm{Plk}^{+/ \Delta 24}$ cells ceased, while divisions in $\mathrm{Plk} 4^{+/ \mathrm{WT}}$ cells proceeded unabated (Fig. 2D). Consistent with the observed reduction in proliferation, $\mathrm{Plk}^{+/ \Delta 24}$ but not Plk4 ${ }^{+/ W T}$ cells showed marked stabilization of p53 and the cell cycle inhibitor p21 $2 \mathrm{~d}$ after infection with adenoviral Cre (Fig. 2E). Centrosome amplification did 
not create a measurable increase in $\gamma \mathrm{H} 2 \mathrm{~A}$.X phosphorylation in Plk4 ${ }^{\Delta 24}$-expressing cells (Fig. 2E), suggesting that DNA damage is unlikely to be the cause of p53 and p21 stabilization in these cells.

The inhibitory effect that loss of Plk4 autoregulation has on cell growth may arise as a consequence of increased levels of Plk4 kinase activity acting on what are as-yet-unidentified substrates. An alternative and nonmutually exclusive possibility is that the extra centrosomes produced as a result of excessive Plk4 levels inhibit long-term cell growth. Since centrosomes are stable structures that persist even after the signal that led to their creation has decayed, inhibition of cellular growth as a result of centrosome amplification should require only a transient elevation in Plk4 levels. To investigate this possibility, we used RPE1 cells in which reversible overexpression of Plk4 ${ }^{\mathrm{AA}}$ can be achieved with a doxycycline-inducible promoter (Wang et al. 2011). Plk4 ${ }^{\mathrm{AA}}$ levels were chronically elevated by continuous treatment with doxycycline or transiently elevated with a 48 -h pulse of

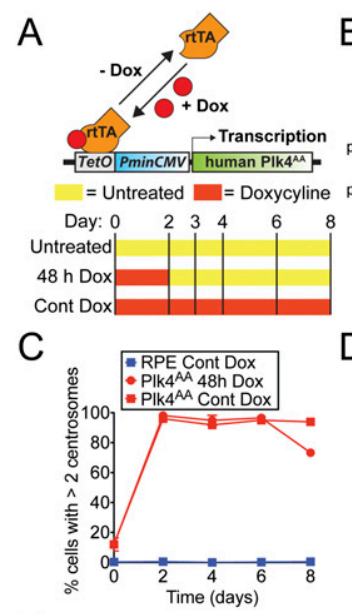

E
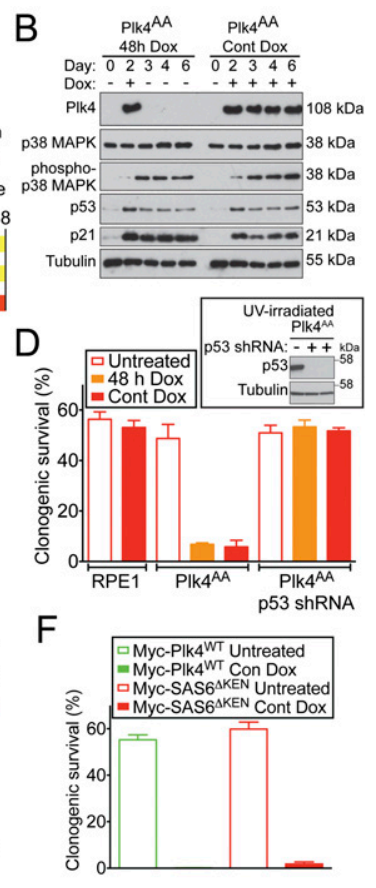

Figure 3. Centrosome amplification inhibits proliferation of RPE1 cells. (A) Schematic of the strategy for doxycycline (Dox)-inducible expression of Plk4 ${ }^{\mathrm{AA}}$ in RPE1 cells. $(B)$ Immunoblots showing the levels of protein in Plk4 $4^{\mathrm{AA}}$ cells at various times after doxycycline addition. Cells were left untreated, grown in the continuous presence of doxycycline, or transiently exposed to doxycycline for $48 \mathrm{~h}$. (C) Fraction of RPE1 and Plk $4{ }^{\mathrm{AA}}$ cells with more than two centrosomes at various times after doxycycline addition. Points show the mean of two independent experiments, and error bars represent the SEM. $(D)$ Percent congenic survival for the indicated cells. Immunoblots of p53 protein in UV-irradiated cells stably expressing a p53 shRNA. Bars represent the mean of two independent experiments carried out in triplicate. Error bars represent the SEM. (E) Fraction of cells with more than two centrosomes at various times after doxycycline addition. Points show the mean of two independent experiments, and error bars represent the SEM. $(F)$ Percent congenic survival for the indicated cells. Bars represent the mean of two independent experiments carried out in triplicate. Error bars represent the SEM. doxycycline (Fig. 3A). Elevated levels of Plk4 ${ }^{\mathrm{AA}}$ declined to below the limit of detection within $<24 \mathrm{~h}$ of removing doxycycline (Fig. 3B). Forty-eight hours of Plk4 $4^{\mathrm{AA}}$ expression drove centrosome amplification in $\sim 100 \%$ of cells, an effect that was maintained even after the disappearance of $\mathrm{Plk} 4^{\mathrm{AA}}$ (Fig. 3C). Increased Plk4 ${ }^{\mathrm{AA}}$ levels and centrosome amplification resulted in activation of $\mathrm{p} 38$ MAPK signaling (as revealed by activating Thr180/ Tyr182 phosphorylation), stabilization of p53, and upregulation of the cyclin-dependent kinase inhibitor $\mathrm{p} 21$ (Fig. 4B). This alteration in cellular homeostasis persisted for $>3$ d after the transiently elevated Plk $4{ }^{\mathrm{AA}}$ was no longer detectable (Fig. 3B). Moreover, the growth rate and clonogenic survival of Plk4 ${ }^{\mathrm{AA}}$ cells were severely impaired whether Plk4 levels were continually or transiently (for $48 \mathrm{~h}$ ) elevated (Fig. 3D; Supplemental Fig. S4A). Essentially identical results were obtained in cells carrying a doxycycline-inducible Plk $4^{\mathrm{WT}}$ transgene (Supplemental Fig. S4B-D). Therefore, even a transient elevation in Plk4 levels is sufficient to bring about a long-term inhibition of cell growth.

As a further test of whether centrosome amplification suppresses the growth of RPE1 cells, we used an alternative means to drive the formation of excessive centrosomes that does not require overexpression of Plk4 and its kinase activity. SAS6 is a conserved structural component required for centriole assembly. Human SAS6 contains a C-terminal KEN box required to target the protein for destruction at the end of mitosis, and expression of SAS6 with a mutated KEN box (SAS6 ${ }^{\Delta \mathrm{KEN}}$ ) has been shown to promote centrosome amplification (Strnad et al. 2007). We created monoclonal RPE1 cells containing either a Myc-SAS $6^{\Delta \mathrm{KEN}}$ or, as a control, a Myc-Plk4 ${ }^{\mathrm{WT}}$ doxycycline-inducible transgene. As expected, overexpression of either Myc-Plk4 ${ }^{\mathrm{WT}}$ or Myc-HsSAS6 ${ }^{\Delta \mathrm{KEN}}$ drove centrosome amplification, but the fraction of cells with excessive centrosome numbers increased more slowly in Myc-HsSAS6 ${ }^{\Delta \mathrm{KEN}}$-expressing cells: More than $85 \%$ of Myc-HsSAS6 ${ }^{\Delta \text { KEN }}$ cells had centrosome amplification at $6 \mathrm{~d}$ after doxycycline addition, while expression of Myc-Plk4 ${ }^{\mathrm{WT}}$ for just $2 \mathrm{~d}$ resulted in $>95 \%$ centrosome amplification (Fig. 3E). Importantly, centrosome amplification resulting from overexpression of Myc-Plk4 ${ }^{\mathrm{WT}}$ or Myc-HsSAS6 ${ }^{\Delta \mathrm{KEN}}$ both caused a dramatic reduction in long-term clonogenic survival (Fig. 3F; Supplemental Fig. S4E). Taken together, these data are consistent with the view that centrosome amplification plays a major role in suppressing the proliferation of cells deficient in Plk4 self-regulation.

We tested whether stabilization of p53 contributes to the prolonged cell cycle delay/arrest that occurs following loss of Plk4 autoregulation and centrosome amplification. Inhibition of p53 through expression of the SV40 large T-antigen completely eliminated the growth defect and reduced clonogenic survival observed following activation of the Plk4 $4^{\Delta 24}$ allele in our gene targeted cell line (Fig. 4A,B; Supplemental Fig. S5A). Clonal cell lines were isolated that stably expressed the SV40 large T-antigen and the Plk $4^{\Delta 24}$ allele. Remarkably, these clones grew indefinitely despite the loss of the autoregulated control of Plk4 levels and dramatic centrosome amplification (Fig. 4C-E; Supplemental Fig. S5B). Preventing p53 accumulation using a stably expressed p53 shRNA (Figs. $3 \mathrm{D}, 4 \mathrm{~F}$ ) also eliminated the reduced clonogenic survival observed after (transient or chronic) doxycycline-induced 


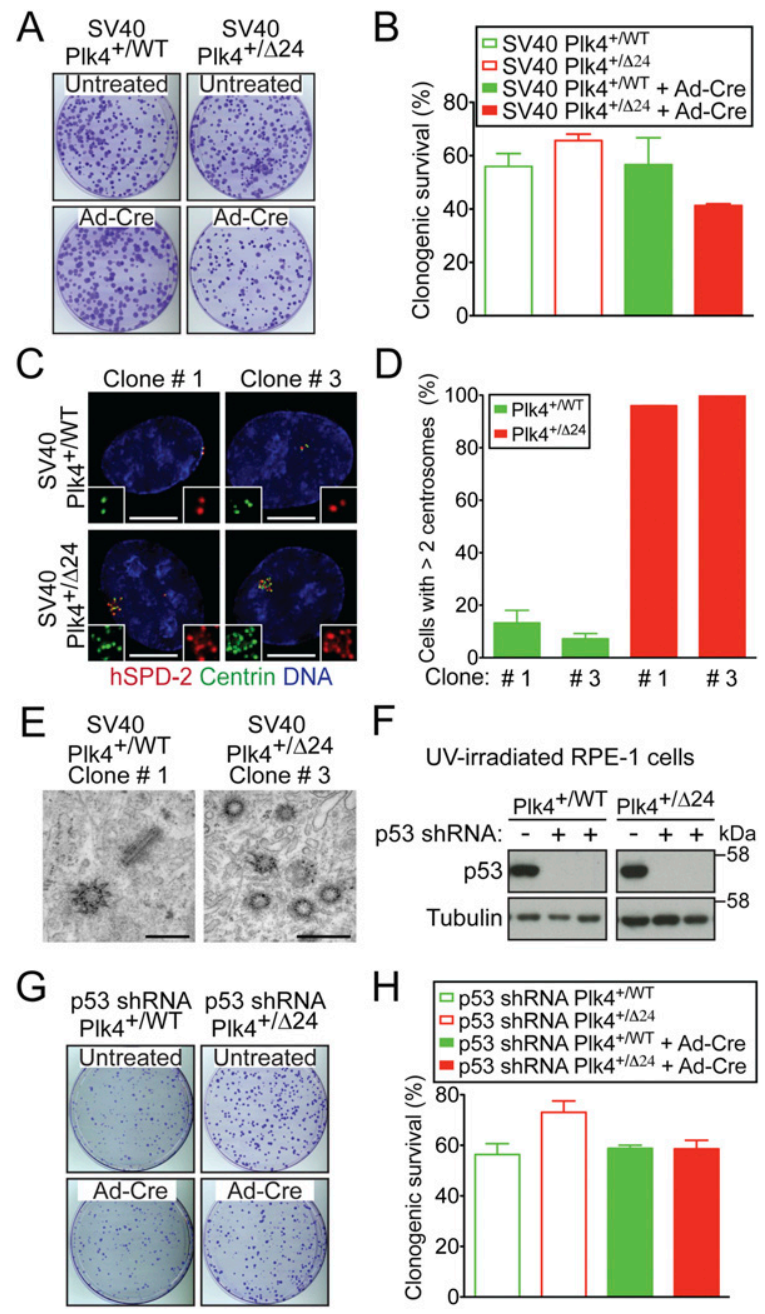

Figure 4. p53 inhibits the proliferation of cells defective in Plk4 autoregulation. (A) Crystal violet-stained colonies of the indicated cell lines. (B) Percent congenic survival for indicated cells. Bars represent the mean of at least two independent experiments carried out in triplicate. Error bars represent the SEM. $(C)$ Images show the level of Plk4 at the centrosome of SV40-RPE1 clones that were isolated after infection with Ad-Cre. (Red) hSPD-2; (green) Centrin; (blue) DNA. Bar, $5 \mu \mathrm{m}$. (D) Fraction of SV40-RPE1 clones with more than two centrosomes. Bars represent the mean of at least two independent experiments, and error bars represent the SEM. (E) Thin-section transmission electron micrographs of SV40-RPE1 clones. Bar, $0.5 \mu \mathrm{m}$. (F) Immunoblots of p53 protein levels in UVirradiated cells stably expressing a p53 shRNA. $(G)$ Crystal violetstained colonies of the indicated cell lines. $(H)$ Percent clonogenic survival for the indicated cells. Bars represent the mean of at least two independent experiments carried out in triplicate. Error bars represent the SEM.

expression of Plk4 ${ }^{\mathrm{AA}}$ (Fig. 3D) or following activation of the Plk $4^{\Delta 24}$ allele in the gene targeted cell line (Fig. 4G,H). Thus, increased Plk4 levels resulting from loss of Plk4 autoregulation bring about a p53-dependent cell cycle arrest.

Here we provide the first evidence that the self-catalyzed destruction of endogenous Plk4 plays a critical role in suppressing Plk4 protein levels to limit centriole duplication to the production of one new centrosome per cell cycle. Preventing Plk4 autoregulation elicits rapid and highly penetrant centrosome amplification followed by a p53-dependent proliferative block. While we cannot formally exclude the possibility that transiently increased Plk4 levels directly suppress cellular proliferation, this would be hard to reconcile with continued growth inhibition after removal of excessive Plk4 and with the block to proliferation caused by increases in the level of SAS6, a structural component of the centrosome. Taken together, our data argue that it is centrosome amplification itself that severely impairs the proliferation of nontransformed RPE1 cells deficient in Plk4 autoregulation. Importantly, removal of p53 overcomes this proliferative block, providing a mechanism for how loss of p53 could lay the foundation for centrosome amplification and further genomic instability.

\section{Materials and methods}

\section{Cell culture and creation of stable cell lines}

hTERT RPE-1 cells were used in all experiments and maintained at $37^{\circ} \mathrm{C}$ in a $5 \% \mathrm{CO}_{2}$ atmosphere with $21 \%$ oxygen. Cells were grown in DMEM:F12 medium containing 10\% tetracycline-free fetal bovine serum (Clontech), $0.348 \%$ sodium bicarbonate, $100 \mathrm{U} / \mathrm{mL}$ penicillin, $100 \mathrm{U} / \mathrm{mL}$ streptomycin, and $2 \mathrm{mM}$ L-glutamine. To carry out Cre recombination, cells were incubated for $4 \mathrm{~h}$ with adenoviral-Cre in DMEM:F12 medium containing $2 \%$ tetracycline-free fetal bovine serum (Clontech), $0.348 \%$ sodium bicarbonate, $100 \mathrm{U} / \mathrm{mL}$ penicillin, $100 \mathrm{U} / \mathrm{mL}$ streptomycin, and 2 mM L-glutamine. Two days after transduction, cells were counted, and the same number of cells in the untreated or Cre-infected population was seeded for growth assays and clonogenic assays.

Stable cell lines expressing RFP-tagged histone H2B, EYFP- $\alpha$-Tubulin, SV40 large T-antigen, or p53 shRNA were created as described (Holland et al. 2012). Doxycycline-inducible Myc-Plk4 and Myc-SAS6 ${ }^{\Delta \text { KEN }}$ hTERT RPE1 cells were created using lentiviral delivery, and single clones were isolated using fluorescence-activated cell sorting. Expression of transgenes was induced with $1 \mu \mathrm{g} / \mathrm{mL}$ doxycycline (Sigma).

\section{Gene targeting}

Gene targeting was performed using adeno-associated virus as previously described (Holland et al. 2012). Taq polymerase (Invitrogen) was used to amplify fragments of the human Plk4 locus from genomic RPE1 DNA for use in construction of the targeting construct. Plk4 AA and $\Delta 24$ mutations were created by QuikChange site-directed mutagenesis (Agilent Technologies). The targeting construct and targeted Plk4 allele were sequenced to verify their integrity.

Detailed Materials and Methods can be found in the Supplemental Material.

\section{Acknowledgments}

We thank Jeffrey Salisbury (Mayo Clinic Foundation, Minnesota) for providing the anti-Centrin antibody, Karen Oegema and Arshad Desai (Ludwig Institute for Cancer Research, California) for providing the human anti-SPD2 and anti-SAS6 antibody, Prasad Jallepalli (Memorial Sloan-Kettering Cancer Center, New York) for providing reagents for AAVmediated gene targeting, Pierre Gönczy (Swiss Federal Institute of Technology, Switzerland) for providing the human SAS6 clone, and Bryan Tsou (Memorial Sloan-Kettering Cancer Center, New York) for providing the doxycycline-inducible Plk4 ${ }^{\mathrm{WT}}$ and Plk4 ${ }^{\mathrm{AA}}$ RPE1 cell lines. We thank Timo Meerloo and Ying Jones of the University of California at San Diego Cellular and Molecular Medicine Electron Microscopy Core Facility for their generous help with electron microscopy, and the University of California at San Diego Neuroscience Microscopy Shared Facility (P30 NS047101). This work was supported by a grant (GM29513) from the National Institutes of Health to D.W.C., who receives salary support from the Ludwig Institute for Cancer Research. A.J.H. is supported by a Leukemia and Lymphoma Society special fellowship. E.A.N. and M.B. gratefully acknowledge support by the Swiss National Science Founda- 
tion (31003A_132428/1). I.M.V. is an American Cancer Society Professor of Molecular Biology and holds the Irwin and Joan Jacobs Chair in Exemplary Life Science. This work was supported in part by a Cancer Center Core Grant (P30 CA014195-38). Q.Z. was supported by a Department of Defense Idea Expansion Award (W81XWH-10-1-0963). D.F. was supported by a European Molecular Biology Organization (EMBO) long-term fellowship.

\section{References}

Basto R, Brunk K, Vinadogrova T, Peel N, Franz A, Khodjakov A, Raff JW. 2008. Centrosome amplification can initiate tumorigenesis in flies. Cell 133: 1032-1042

Bettencourt-Dias M, Rodrigues-Martins A, Carpenter L, Riparbelli M, Lehmann L, Gatt MK, Carmo N, Balloux F, Callaini G, Glover DM. 2005. SAK/PLK4 is required for centriole duplication and flagella development. Curr Biol 15: 2199-2207.

Brownlee CW, Klebba JE, Buster DW, Rogers GC. 2011. The protein phosphatase $2 \mathrm{~A}$ regulatory subunit Twins stabilizes Plk4 to induce centriole amplification. I Cell Biol 195: 231-243.

Cunha-Ferreira I, Rodrigues-Martins A, Bento I, Riparbelli M, Zhang W, Laue E, Callaini G, Glover DM, Bettencourt-Dias M. 2009. The SCF/ Slimb ubiquitin ligase limits centrosome amplification through degradation of SAK/PLK4. Curr Biol 19: 43-49.

Ganem NJ, Godinho SA, Pellman D. 2009. A mechanism linking extra centrosomes to chromosomal instability. Nature 460: 278-282.

Gerber SA, Rush J, Stemman O, Kirschner MW, Gygi SP. 2003. Absolute quantification of proteins and phosphoproteins from cell lysates by tandem MS. Proc Natl Acad Sci 100: 6940-6945.

Gonczy P. 2012. Towards a molecular architecture of centriole assembly. Nat Rev Mol Cell Biol 13: 425-435.

Guderian G, Westendorf J, Uldschmid A, Nigg EA. 2010. Plk4 transautophosphorylation regulates centriole number by controlling BTrCP-mediated degradation. J Cell Sci 123: 2163-2169.

Habedanck R, Stierhof YD, Wilkinson CJ, Nigg EA. 2005. The Polo kinase Plk4 functions in centriole duplication. Nat Cell Biol 7: 1140-1146.

Holland AJ, Cleveland DW. 2012. Losing balance: The origin and impact of aneuploidy in cancer. EMBO Rep 13: 501-514.

Holland AJ, Lan W, Cleveland DW. 2010a. Centriole duplication: A lesson in self-control. Cell Cycle 9: 2731-2736.

Holland AJ, Lan W, Niessen S, Hoover H, Cleveland DW. 2010b. Polo-like kinase 4 kinase activity limits centrosome overduplication by autoregulating its own stability. J Cell Biol 188: 191-198.

Holland AJ, Fachinetti D, Da Cruz S, Zhu Q, Vitre B, Lince-Faria M, Chen D, Parish N, Verma IM, Bettencourt-Dias M, et al. 2012. Polo-like kinase 4 controls centriole duplication but does not directly regulate cytokinesis. Mol Biol Cell 23: 1838-1845.

Kleylein-Sohn J, Westendorf J, Le Clech M, Habedanck R, Stierhof YD, Nigg EA. 2007. Plk4-induced centriole biogenesis in human cells. Dev Cell 13: 190-202.

Ko MA, Rosario CO, Hudson JW, Kulkarni S, Pollett A, Dennis JW, Swallow CJ. 2005. Plk4 haploinsufficiency causes mitotic infidelity and carcinogenesis. Nat Genet 37: 883-888.

Liu L, Zhang CZ, Cai M, Fu J, Chen GG, Yun J. 2012. Downregulation of polo-like kinase 4 in hepatocellular carcinoma associates with poor prognosis. PLOS ONE 7: e41293. doi: 10.1371/journal.pone.0041293.

Macmillan JC, Hudson JW, Bull S, Dennis JW, Swallow CJ. 2001. Comparative expression of the mitotic regulators SAK and PLK in colorectal cancer. Ann Surg Oncol 8: 729-740.

Nigg EA, Raff JW. 2009. Centrioles, centrosomes, and cilia in health and disease. Cell 139: 663-678.

Peel N, Stevens NR, Basto R, Raff JW. 2007. Overexpressing centriolereplication proteins in vivo induces centriole overduplication and de novo formation. Curr Biol 17: 834-843.

Rogers GC, Rusan NM, Roberts DM, Peifer M, Rogers SL. 2009. The SCF Slimb ubiquitin ligase regulates Plk4/Sak levels to block centriole reduplication. J Cell Biol 184: 225-239.

Silkworth WT, Nardi IK, Scholl LM, Cimini D. 2009. Multipolar spindle pole coalescence is a major source of kinetochore mis-attachment and chromosome mis-segregation in cancer cells. PLOS ONE 4: e6564. doi: 10.1371/journal.pone.0006564.

Sillibourne JE, Tack F, Vloemans N, Boeckx A, Thambirajah S, Bonnet $\mathrm{P}$ Ramaekers FC, Bornens M, Grand-Perret T. 2010. Autophosphoryla- tion of polo-like kinase 4 and its role in centriole duplication. $\mathrm{Mol}$ Biol Cell 21: 547-561.

Strnad P, Leidel S, Vinogradova T, Euteneuer U, Khodjakov A, Gonczy P. 2007. Regulated HsSAS-6 levels ensure formation of a single procentriole per centriole during the centrosome duplication cycle. Dev Cell 13: 203-213.

Tsou MF, Stearns T. 2006. Controlling centrosome number: Licenses and blocks. Curr Opin Cell Biol 18: 74-78.

Wang WJ, Soni RK, Uryu K, Tsou MF. 2011. The conversion of centrioles to centrosomes: Essential coupling of duplication with segregation. J Cell Biol 193: 727-739. 


\section{Erratum}

Genes \& Development 26: 2684-2689 (2012)

The autoregulated instability of Polo-like kinase 4 limits centrosome duplication to once per cell cycle Andrew J. Holland, Daniele Fachinetti, Quan Zhu, Manuel Bauer, Inder M. Verma, Erich A. Nigg, and Don W. Cleveland

After review of the above-mentioned article, the authors added an immunoblot panel to Figure 2E that resulted in the accidental duplication of the "p53 pSer15" immunoblot on top of the "p21" immunoblot. The corrected Figure 2E, containing the original "p21" immunoblot that was obscured in the published image, is shown below and in the article PDF on the journal website, which has been updated to reflect this change. The authors apologize for any inconvenience this may cause.

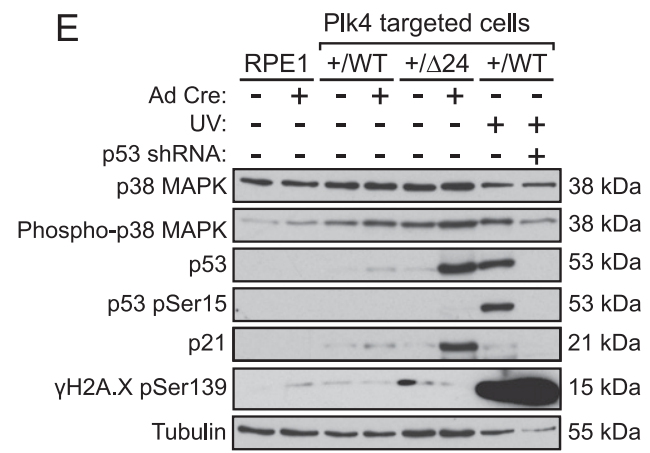




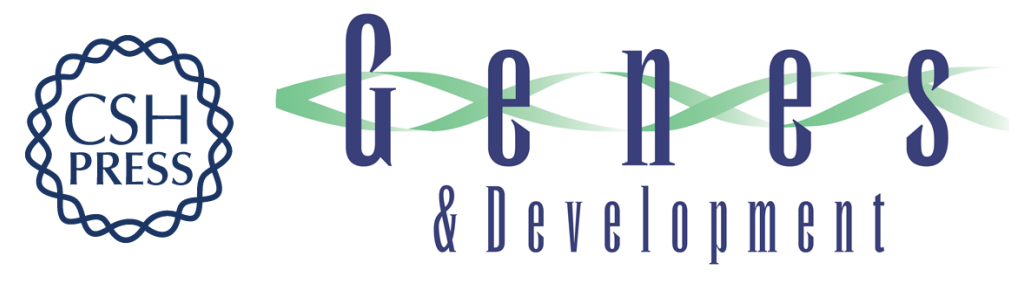

\section{The autoregulated instability of Polo-like kinase 4 limits centrosome duplication to once per cell cycle}

Andrew J. Holland, Daniele Fachinetti, Quan Zhu, et al.

Genes Dev. 2012, 26:

Access the most recent version at doi:10.1101/gad.207027.112

\section{Supplemental http://genesdev.cshlp.org/content/suppl/2012/12/19/26.24.2684.DC1 \\ Material}

Related Content

The autoregulated instability of Polo-like kinase 4 limits centrosome duplication to once per cell cycle

Andrew J. Holland, Daniele Fachinetti, Quan Zhu, et al.

Genes Dev. January, 2013 27: 226

References This article cites 25 articles, 8 of which can be accessed free at:

http://genesdev.cshlp.org/content/26/24/2684.full.html\#ref-list-1

Articles cited in:

http://genesdev.cshlp.org/content/26/24/2684.full.html\#related-urls

\section{License}

Email Alerting

Service

Receive free email alerts when new articles cite this article - sign up in the box at the top right corner of the article or click here.

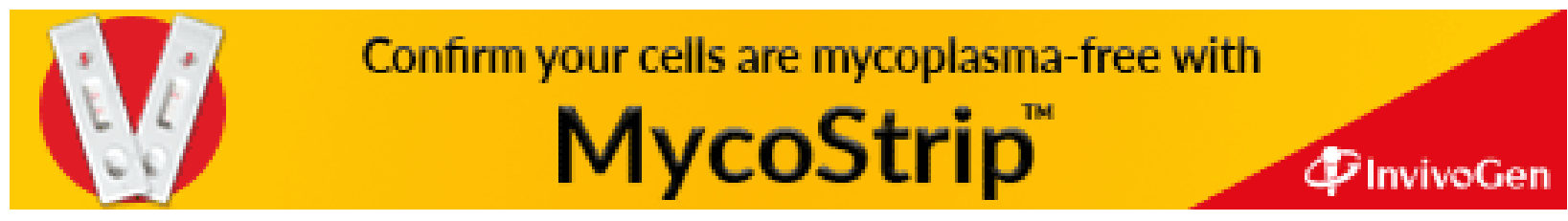

